\title{
A Finite Pool of Worry or a Finite Pool of Attention? Evidence and Qualifications
}

Matthew Sisco ( $\sim$ ms4403@columbia.edu )

Columbia University

Sara Constantino

Princeton University

Yu Gao

Peking University https://orcid.org/0000-0001-7519-0062

Massimo Tavoni

European Institute on Economics and the Environment

Alicia Cooperman

Texas A\&M University https://orcid.org/0000-0002-1652-4488

Valentina Bosetti

Bocconi University https://orcid.org/0000-0003-4970-0027

Elke Weber

Princeton University

Article

Keywords: Finite Pool of Worry, COVID-19, affect generalization, big data

Posted Date: November 2nd, 2020

DOI: https://doi.org/10.21203/rs.3.rs-98481/v1

License: (c) (1) This work is licensed under a Creative Commons Attribution 4.0 International License.

Read Full License 


\section{Abstract}

The Finite Pool of Worry (FPW) hypothesis states that humans have finite emotional resources for worry, so that when we become more worried about one threat, it can decrease worry about other threats.

Despite its relevance, no conclusive empirical evidence for the hypothesis exists. We leverage the sudden onset of new worries introduced by the COVID-19 pandemic as a natural experiment to test the FPW. In six metropolitan areas across three countries (USA, Italy, and China) we assessed social media attention, news attention, self-reported attention, and self-reported worries about various threats (climate change, terrorism, economy, and unemployment) throughout the pandemic. As attention to and worry about COVID-19 increased, we find that attention to climate change decreased but worry about it did not. Results are confirmed by further analysis with a large, and nationally representative U.S. sample. We find some perceived similarity between COVID-19 and climate change, but this does not fully explain the positive relationship in worry we see between them. We also find that more negative personal experience with COVID-19 is positively associated with climate change worry even while controlling for relevant covariates. We lastly examine the aggregate effect of COVID-19 worry on support for climate policies and find that greater COVID-19 worry is associated with more cross-partisan support for climate change policies, even when controlling for political ideology and other covariates. In summary, our findings suggest that while there appears to be a Finite Pool of Attention to threats, we do not see evidence of a Finite Pool of Worry.

\section{Full Text}

Due to technical limitations, full-text HTML conversion of this manuscript could not be completed. However, the latest manuscript can be downloaded and accessed as a PDF.

\section{Figures}


COVID-19 Social Media Attention vs. Other Worries
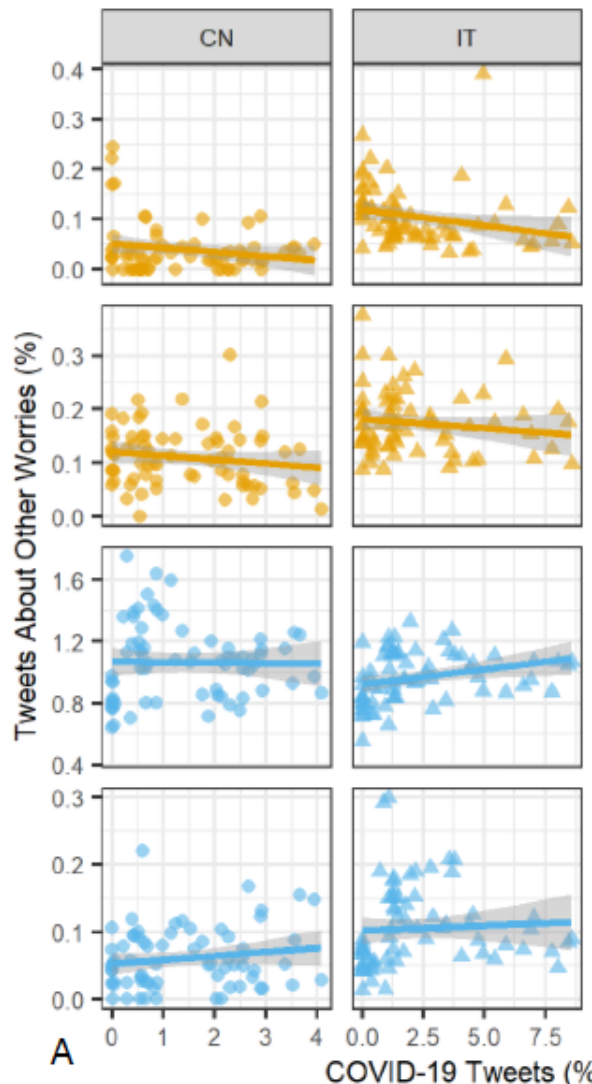

COVID-19 Tweets (\%)
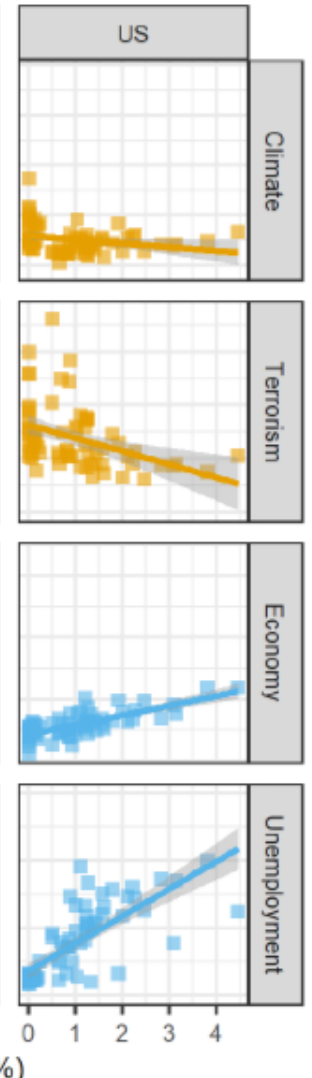

COVID-19 News Attention vs. Other Worries
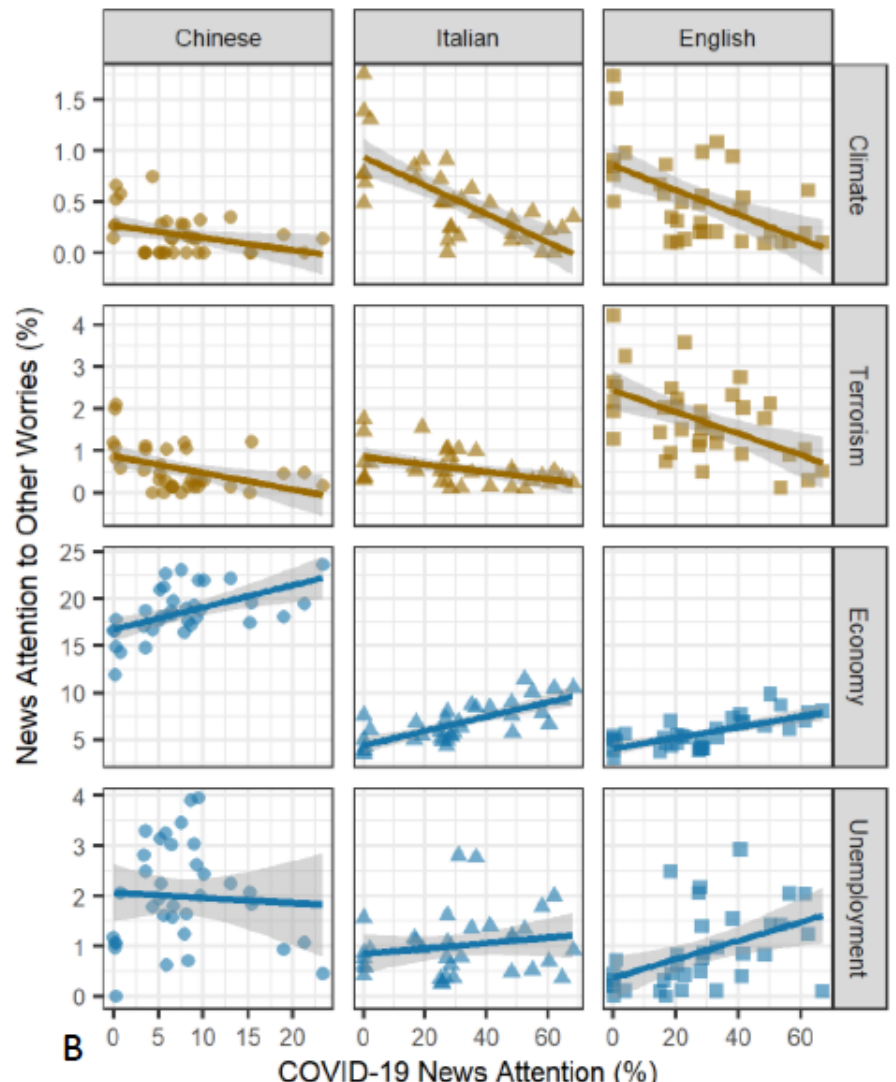

\section{Figure 1}

COVID-19 attention on social media and news media crowds out attention to climate and terrorism. Units are percentages of all social media messages / news articles aggregated weekly. Data points in panel A are aggregated by city and in panel $B$ aggregated by language. The lines show the bivariate regression fits and the shaded regions show the $95 \%$ Cls. 


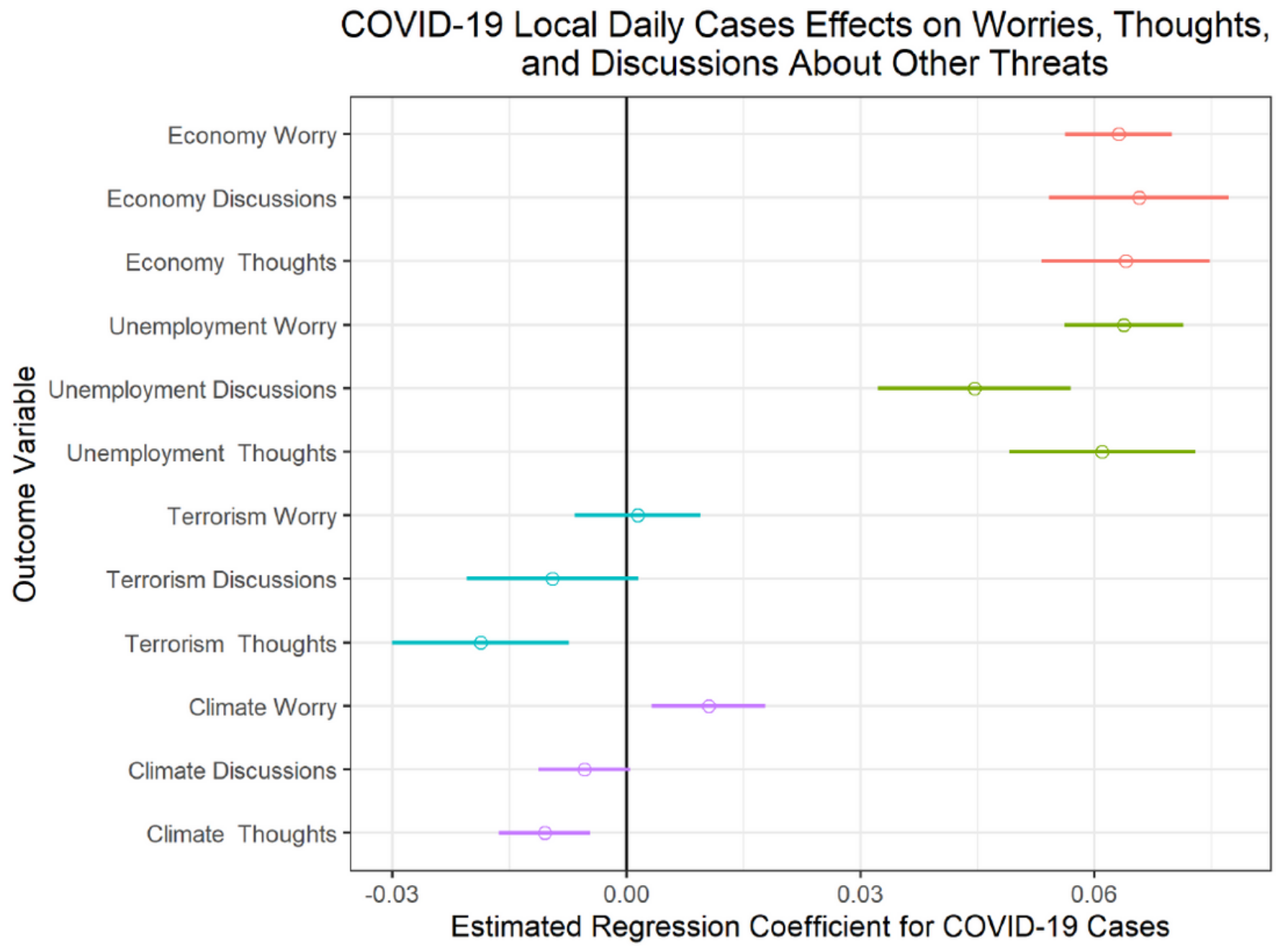

Figure 2

Worries, discussions, and thoughts about four threats regressed on (log) COVID-19 regional cases. Each value corresponds to a beta coefficient estimate for (log) COVID-19 cases predicting one of the twelve outcome variables shown in each row. The full regression table with estimates for all explanatory and outcome variables is shown in Supplemental Table 1. Error bars depict 95\% Cls. 


\section{Climate Worry vs. Change in COVID-19 Worry}

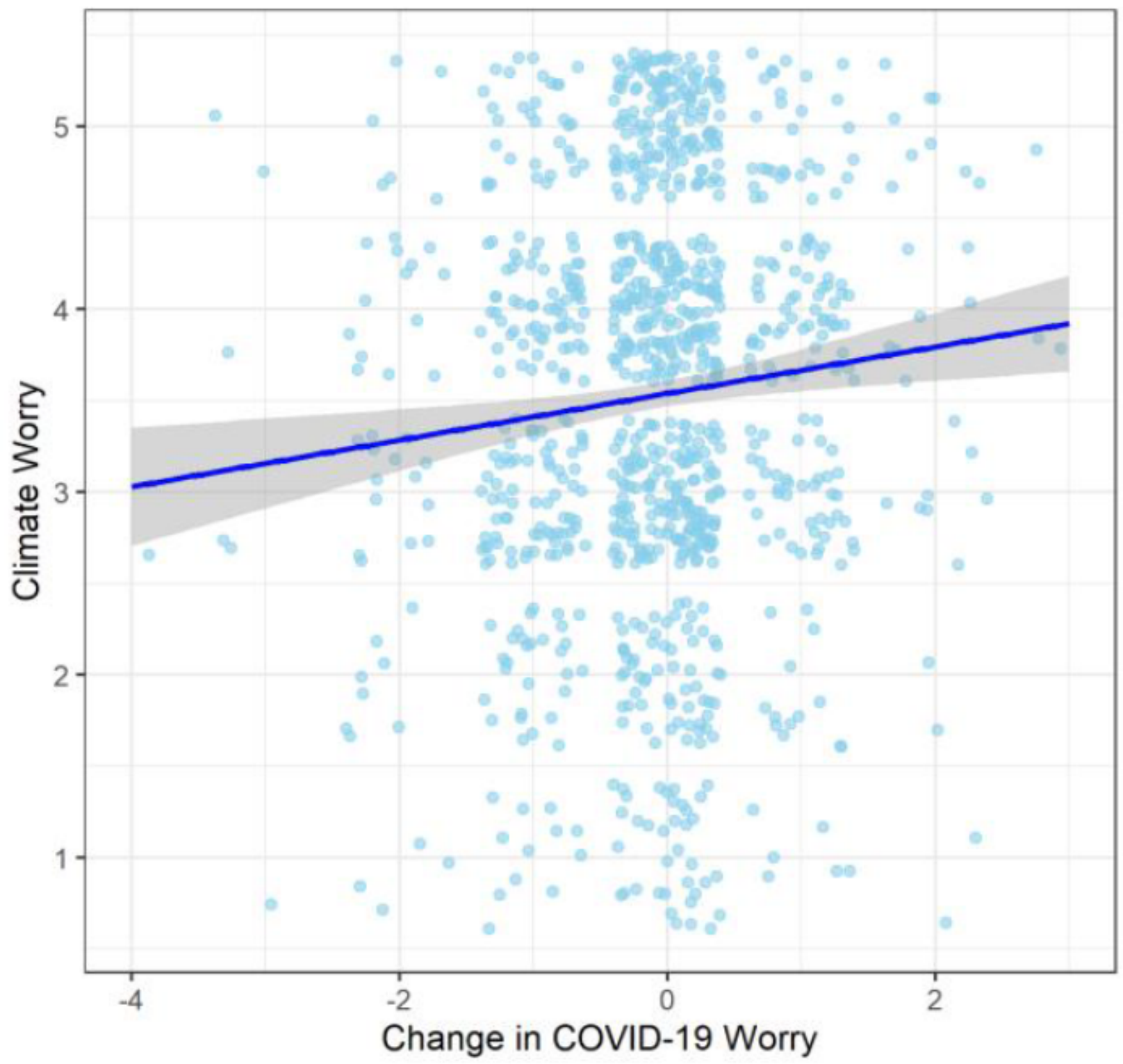

Figure 3

Climate worry and within-person change in COVID-19 worry. Climate worry shown here is measured on participants' second survey response (approx. one month after their first responses). The line shown is a bi-variate regression fit and the shaded region shows the $95 \%$ confidence interval. 


\section{COVID-19 effects on Climate Worry}

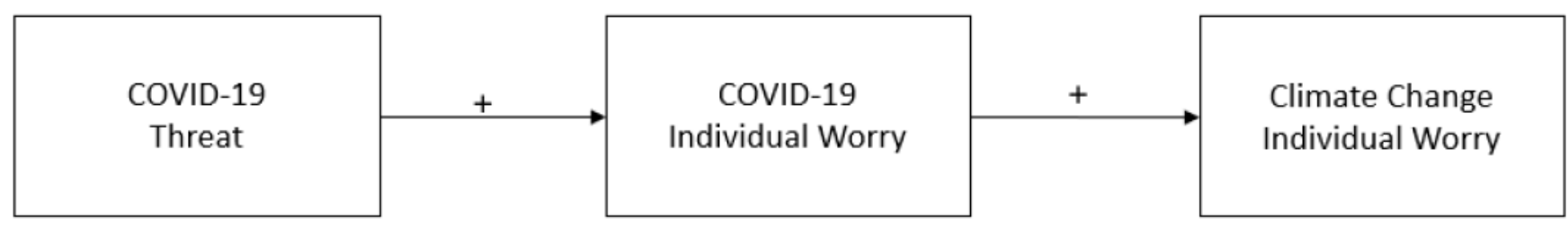

COVID-19 Effects on Climate Attention

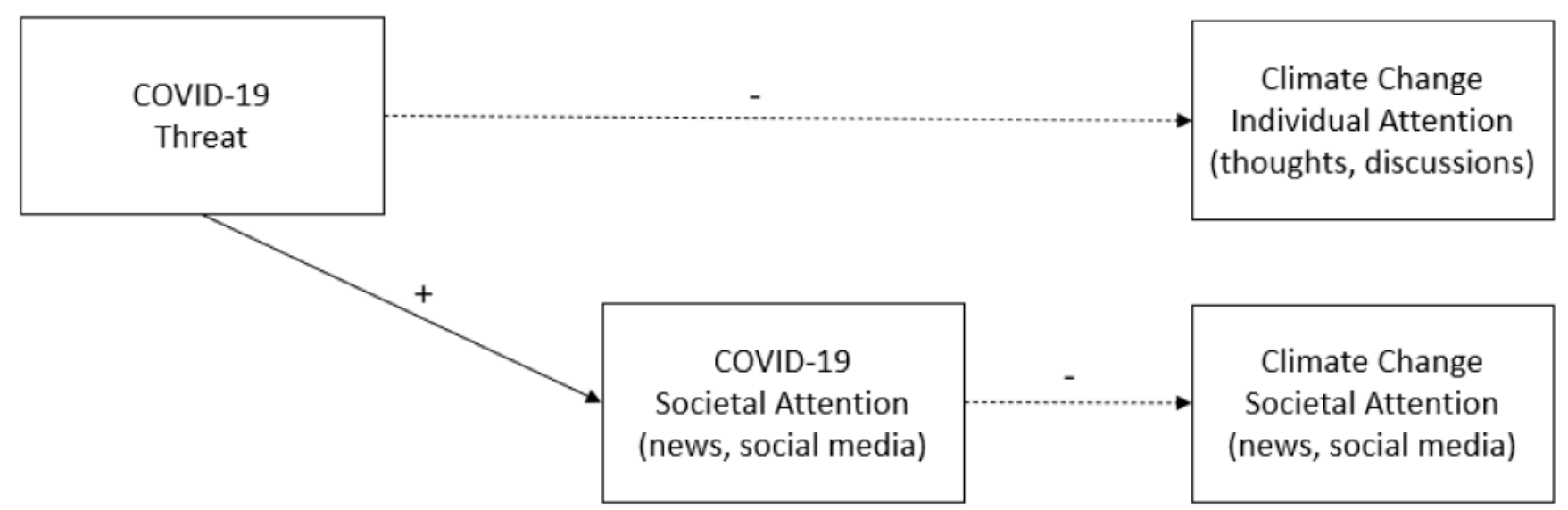

\section{Figure 4}

Conceptual diagram summarizing the main results of the studies presented here. Plus or minus signs indicate a positive or negative relationship between two constructs.

\section{Supplementary Files}

This is a list of supplementary files associated with this preprint. Click to download.

- AFinitePoolofWorryoraFinitePoolofAttentionSupplementaryMaterials.pdf 